\title{
Reliability Research of the Traffic Signal System Based on Extended Petri Nets
}

\author{
YaQin Song ${ }^{1}$, Bing $\mathrm{Li}^{2}$, Keli Chen, Tao Yang and Honge Li \\ School of Mathematics and Computer Engineering, Xihua University, \\ Chengdu, China \\ 1864530116@qq.com, 2ibing_lyl@163.com
}

\begin{abstract}
The reliability of the traffic signal in the intersection is extremely important to the safe and orderly urban traffic. In this paper, basing on the Classics Petri Nets, we put forward a kind of Extended Colored Petri Nets with the inhibitory and enable arc, so we built the traffic control model based on the improved Petri Nets to ensure the safe and orderly traffic; the model can reflect the dynamic process of intersection and realize the detection and processing of "green conflict". From the angle of logical equivalence, we use digital logic circuit to simulate the traffic model which is built based on the Extended Colored Petri Nets with the inhibitory and enable arc. The simulation results show that the model based on improved Petri Nets can effectively detect and adjust "green conflict" together with other potential safety risks; also verify the validity of the Extended Colored Petri Nets and the traffic control model.
\end{abstract}

Keywords: Reliability of traffic signal, Extended Colored Petri Nets, green conflict, modeling and verifying

\section{Introduction}

With the acceleration of urbanization in recent years, the structure of road network becomes more and more complex, how to make full use of road resource and to ensure effective traffic is particularly important. With new theories and technologies applied in the traffic control system increasingly, groups of efficient traffic control system are formed. The traffic safety is the primary factor, therefore the reliability of the traffic control system is very important; reliable traffic control signal system is primary way to take full advantage of the road resource and ensure the safety and order of traffic.

In the traffic control system, Petri Nets model not only can conveniently describe the synergy, competitiveness and synchronization, it also may change the structure of nets, the logic, opportunity, harmony of the traffic system, and the assumed input stream[1]. Therefore, it is feasible to model traffic control system by Petri Nets. On the one hand, it can reflect the variable process of the traffic flow under normal circumstance; on the other hand, it can realize the detection of "green conflict" and regulate the system to the right phase automatically, then guarantee the safety of the intersection.

While, the traditional Petri Nets cannot describe the data stream accurately; and lack of hierarchy system, so people introduced Advanced Petri Nets on the basis of Classics Petri Nets, such as the Color Petri Nets proposed in the reference [3, 4], it is fully capable to describing the data through classifying for tokens, in this way it could realize the folding of the net system and then reduce its complexity. Even though, the Color Petri Nets is not better than the Classics Petri Nets in simulation capability, it cannot model the systems with special characters. In order to improve the simulation capability, the Extended Petri Nets was introduced on the basis of Advanced Petri Nets, it can enhance the simulation ability by increasing the elements of the net system, such as the Petri Nets with inhibitory-arc and enable-arc proposed in reference [7], the inhibitory-arc can control the 
transition's order of occurrence, and describe the precedence relationship between events. So this kind of Petri Nets is able to describe the system that has the property of priority. In this paper we use the Place to represent data and control information; the inhibitory-arc can strengthen the simulation capability to the priority of events. Finally, the Extended Colored Petri Nets with the Inhibitory and Enable Arc can describe the system with priority, at the same time, and it also can make the model more clear and concise.

\section{Extended Colored Petri Nets with the Inhibitory and Enabling Arc}

\subsection{Syntactic Structure}

Definition1. $\bullet t=\{p \in P \mid(p, t) \in F\}$, it denotes the common pre-set of transitions called t. $t^{\bullet}=\{p \in P \mid(t, p) \in F\}$, it denotes the common post-set of transitions called t. $t_{I}=\{p \in P \mid(p, t) \in I\}$, it denotes the inhibitory set of transitions called $\mathrm{t}$. $t_{E}=\{p \in P \mid(p, t) \in E\}$, it denotes the enabling set of transitions called t.

Definition2. The Petri Net with the Inhibitory and Enabling Arc is a six tuple $\sum=(P, T ; F, I, E, M)$.

$N=(P, T ; F)$ denotes a net, $M$ represents the current mark of the net system, $I \subset P \times T$ represents finite set of inhibitor arcs, $E \subset P \times T$ represents finite set of enable arcs; and they satisfy the relationship $(I \cup E) \cap F=\varnothing$ (namely, $\forall p \in P \wedge \forall t \in T:(p, t) \in F \rightarrow(p, t) \notin I)$.

1) $\quad t \in T$

A. If what points to it is inhibitory arcs and satisfies the following two conditions:

a) $\forall p \in P:(p, t) \in F \rightarrow M(p) \geq 1$

b) $\forall p \in P:(p, t) \in I \rightarrow M(p)=0$

So the transition t marked currently by $\mathrm{M}$ would occur, written as $M[t>$.

B. If what points to it is enabling arcs and satisfies the following two conditions:

a) $\forall p \in P:(p, t) \in F \rightarrow M(p) \geq 1$

b) $\forall p \in P:(p, t) \in E \rightarrow M(p) \geq 1$

So the transition t marked currently by $\mathrm{M}$ would occur, written as $M[t>$.

2) If $M\left[t>\right.$, transition $t$ would change the mark $M$ into a new mark $M^{\prime}$ :

$$
M^{\prime}=\left\{\begin{array}{l}
M(p)-1, \text { if }(p, t) \in F \wedge(t, p) \notin F \\
M(p)+1, \text { if }(t, p) \in F \wedge(p, t) \notin F \\
M(p), \text { others }
\end{array}\right.
$$

From this formula, we know that the differences of the transition occurring conditions between the inhibitory and enable arcs embody at the formulas (2) and (4). The formula (5) conveys that the changes of current mark are same as the circumstance that delete the inhibitory and enable arcs from the net systems.

From the definition.2, we see that the inhibitory and enable arcs is just play a control role in whether the transition with occurring condition occurred in the prototype Petri nets sense, once it occurred, they do not influence the current mark changing caused by it. We can use them to describe the system that needs the judge of priority right and resolve the randomness of the transition occurring in Petri nets [2]. For example, in the fig.3, the inhibitory arc derived from the Place of "green conflicts" $P_{g}$ made the system to firstly judge whether some transition of $T g 1 \sim T g 8$ is triggered or not, and then judges whether the 
transitions which represent the vehicles' motion(straight on or turning left) of each intersection are triggered or not.

Definition3. Extended Colored Petri Nets with The Inhibitory and Enable Arc is a ten-tuple $\sum=\left(P, T ; F, C, W_{F}, I, W_{I}, E, W_{E}, M\right)$.

Among it,

(1) $N=(P, T ; F)$ Represents a net.

(2) “ $C$ " represents a finite set of color; and $C=\left\{c_{1}, c_{2}, c_{3}, c_{4} \cdots \cdots c_{k}\right\}$.

(3) $W_{F}: F \rightarrow L(C)_{+}$Denotes a mapping relationship from a finite set to the colors set.

(4) $I \subset P \times T$ Represents a finite set of inhibitory arcs. $E \subset P \times T$ represents finite set of enable arcs, and $I \cap E=\varnothing,(I \cup E) \cap F=\varnothing$.

(5) $W_{I}: I \rightarrow C^{\prime}\left(C^{\prime} \subseteq C\right)$ Denotes a mapping relationship from the inhibitory set $I$ to the proper subset $\mathrm{C}^{\prime}$ of the colors set $\mathrm{C}$.

(6) $M: P \rightarrow L(C)$ Denotes a mapping relationship from the Place to the color domain function.

In the above definition, $W_{F}, W_{I}$ and $W_{E}$ denote the weight functions that are defined in the finite arc set $F$, the inhibitory arc set $I$ and the enabling arc set $E$ respectively. $L(C)$ is Non-negative integer coefficient linear function defined in the colors set $C . L(C)_{+}$is a sub-set of $L(C)$, its coefficients are not all zero.

1) $t \in T$

A. If what points to it is inhibitory arcs and satisfied the following two conditions:

$$
\begin{aligned}
& \text { a) } \forall p \in P:(p, t) \in F \rightarrow M(p) \geq W_{F}(p, t) \\
& \text { b) } \forall p \in P:(p, t) \in I \rightarrow L^{\prime}(M(p)) \cap W_{I}(C)=\varnothing
\end{aligned}
$$

So transition t marked currently by $\mathrm{M}$ would occur, written as $M[t>$.

B. If what points to it is enabling arcs and satisfied the following two conditions:

$$
\begin{aligned}
& \text { a) } \forall p \in P:(p, t) \in F \rightarrow M(p) \geq W_{F}(p, t) \\
& \text { b) } \forall p \in P:(p, t) \in E \rightarrow L^{\prime}(M(p)) \supseteq W_{E}(C)
\end{aligned}
$$

So transition t marked currently by $\mathrm{M}$ would occur, written as $M[t>$.

Among the above definition, $\left(L^{\prime}(M(p)) \subseteq C\right)$, namely, $L^{\prime}(M(p))$ represents a set composed by elements $\mathrm{C}_{K}\left(\mathrm{C}_{\mathrm{K}} \in \mathrm{C}\right)$; and $\mathrm{C}_{\mathrm{K}}\left(\mathrm{C}_{\mathrm{K}} \in \mathrm{C}\right)$ represent non-zero coefficients of linear equation of $M(p)$.

3) If $M\left[t>\right.$, transition $t$ would change the mark $M$ into a new mark $M^{\prime}$ :

$$
M^{\prime}=\left\{\begin{array}{c}
M(p)-W_{F}(p, t), p \in t-t \\
M(p)+W_{F}(p, t), p \in t-t \\
M(p)+W_{F}(p, t)-W_{F}(p, t), p \in t-t \\
M(p), \text { others }
\end{array}\right.
$$

From the formula (7), we can draw such a conclusion, when the transitions connect with the inhibitory arcs, the transitions may occur just only as there is no intersection between the colors set on the inhibitory arcs and the colors sets on the Place which is connected. Similarly, from the formula (9), we can draw such a conclusion also, when the transitions connect with the enabling arcs, the transitions may occur just only as the colors set on the enabling arcs is contained in the colors set on the Place which is connected.

From the definition.3, we can draw the following conclusions. Comparing it with Classics Petri Nets, the Extended Colored Petri Nets with the Inhibitory and Enabling Arc 
have an abundant weight functions on their Arcs and tokens on their Place, they also can include a variety of colors. Even though the definition of this kind of Petri Net is complicated, it can make the complex system modeling brief and clear to a certain extent. Such as the Figure 3, the colors set on the Place $P_{E}$ is $C=\left\{S, L, C_{l}\right\}$, it needs two Places to respectively describe the green lights of straight-on or turning left at eastern crossroad if by Classics Petri Nets, nevertheless, only one Place can solve this problem by the Extended Colored Petri Nets, so in this way the complexity of the model is reduced extremely.

\subsection{Graphical Notations}

The Extended Colored Petri Nets with Inhibitory Arc and Enabling Arc can be described by directed graph with weight factor. Graphic elements include Place, transition and arcs; and arcs include Inhibitory Arc, Enabling Arc and directed Arc. Place is the element that describe state, it is represented with a circle, the different colors of token in the Place represent different objects or information; Transition is the element that describe variation, it is represented with rectangle; the weight functions on the directed arc are described by linear expression of colors set; the arc derived from Place to Transition and a hollow dot on arc denote the inhibitory-arc, the arc derived from Place to Transition and a solid dot on arc denote the enable-arc, a subset of colors set to denotes the weight function on the inhibitory-arc and enable-arc. As the Fig.1 shows, it describes a brief Extended Colored Petri Nets with The Inhibitory and Enable Arc.

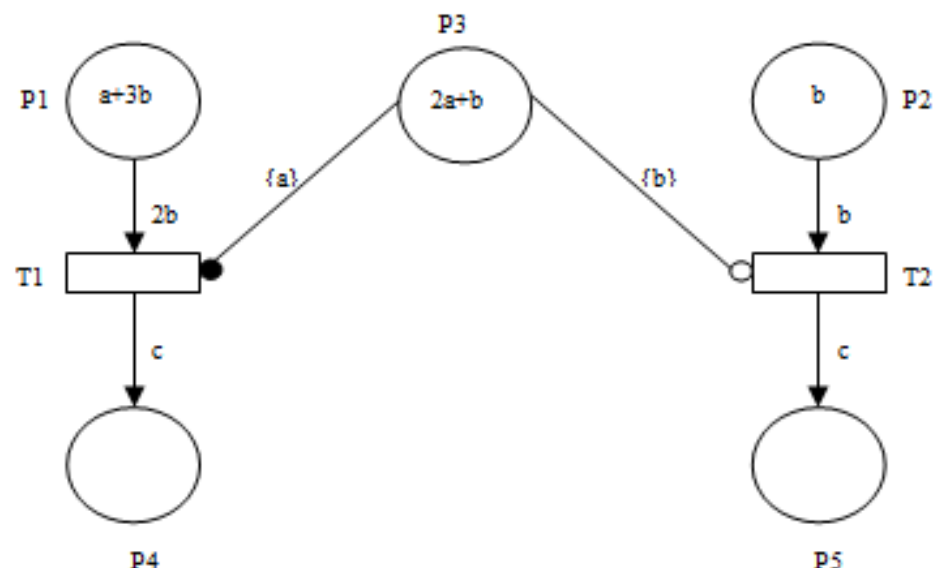

Figure 1. Extended Colored Petri Nets with the Inhibitory and Enable Arc

In the above-mentioned figure, the transition $T l$ 's pre-set $\cdot t$ is $P 1$ and its post-set $t$. is $P 4$, the Place $P 3$ is connected with the enable-arc. On the current mark, the token of Place $P 1$ is $M(P 1)=a+3 b$, the weight function of the directed arc from the Place $P 1$ to the transition $T 1$ is $W_{F}(P 1, T 1)=2 b$, therefore $M(P 1) \geq W_{F}(P 1, T 1)$; while the token of Place $P 3$ that is connected with the transition $T 1$ through an enable-arc is $M(P 3)=2 a+b$, from it we know that $L^{\prime}(M(P 3))=\{a, b\}$, at the same time $W_{E}(C)=\{a\}$, so $L^{\prime}(M(P 3)) \supseteq W_{E}(C)$ is established. In conclusion, the transition $T 1$ can occur. Once the transition $T 1$ occurred, the token of the Place $P 1$ would change to $M(P 1)=a+b$, at the same time the token of $P 4$ would change to $M(P 4)=c$, however the token of $P 3$ would not change. We can conclude that the occurrence of a transition usually just involved some adjacent and not all tokens of the Places. Similarly, according to triggering condition of the transition described in definition 3, the transition $T 2$ does not own occurring right. 


\subsection{Model Simulation}

The "green conflict" detection model is basing on a Extended Petri Nets-—Colored Petri Nets with The Inhibitory and Enable Arc in this paper. Because it extend the definition of the traditional Petri Nets, so the existed simulation tools such as CPN Tools and Tina that used for the traditional Petri Nets model simulation cannot verify the extended Petri Nets model. Basing on logic equivalence principle, we adopt digital logic hardware circuit to analysis the model. There are three elements in the Petri Nets: Place, Transition and Arc. The Place is used for storing token, so we can use trigger to realize it; the Transition can be replaced by logic gates; the wire can be replaced by the Arc. In this way, each element in the Petri Nets is mapped to the corresponding digital logic hardware circuit. So the digital logic circuit can simulate the model. If we assign initial value to logic circuit, the working process of the circuit system is same as the operation of the Petri Nets, the output of the circuit system would be the final result of the model, and thus we use digital logic circuit to imitate the running process of the Petri Nets model.

\section{Modeling and Verifying of the Traffic Control Signal System}

\subsection{Model of the System}

Naturally, vehicles would be allowed to pass only one direction of crossroad at a given time. But, "green conflict" and other abnormalities may happen when the traffic control signal system gets disturbed. It may result in traffic accidents and even threat to the safety of the people if we cannot detect or solve these problems. Therefore it is significant to detect and conduct "green conflict" and other abnormalities. In this paper we build a model of the common crossroads to realize detection and adjustment of the "green conflict".

Under normal circumstances, the control system can only run one green light phase at one moment. The control system perhaps comes into being "green conflict" because of outside interference or system software reliability, the vehicles' movement direction would be perpendicular at a given time, this phenomenon can be explained by the following example. There is a crossroad; A, B, C and D respectively represent its east, south, west and north, AC represents straight-on direction from east to west, while CA represents straight-on direction from west to east, other and so on. Now, there are six green-conflicting states with $\mathrm{AC}$, these states include $\mathrm{BD}, \mathrm{DB}, \mathrm{CD}$ (left-turn), $\mathrm{DA}($ left-turn), $\mathrm{BC}$ (left-turn) and $\mathrm{AB}$ (left-turn). Among them, $\mathrm{BC}$ (left-turn) and $\mathrm{AB}$ (left-turn) are perhaps green-conflicting with $\mathrm{AC}$ indirectly if $\mathrm{AC}$ direction and $\mathrm{CA}$ direction are both allowed at the same time; while "green conflict" with $\mathrm{BD}$ (not including the state that green-conflict with $\mathrm{AC}$ ) includes $\mathrm{DA}$ (left-turn), $\mathrm{AB}$ (left-turn) and $\mathrm{CD}$ (left-turn). Usually the traffic of crossroad is controlled by 4-phase signal or 8-phase signal. For general crossroad, 4-phase signal will be able to ensure a high traffic rate so we choose 4-phase. Figure 2 shows a crossroad traffic control system by 4-phase. 




(a) phase-1

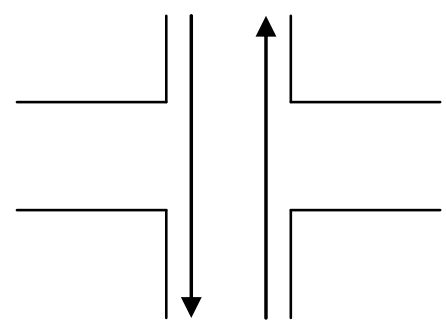

(c) phase-3

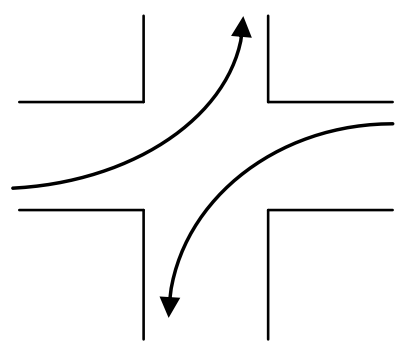

(b) phase-2

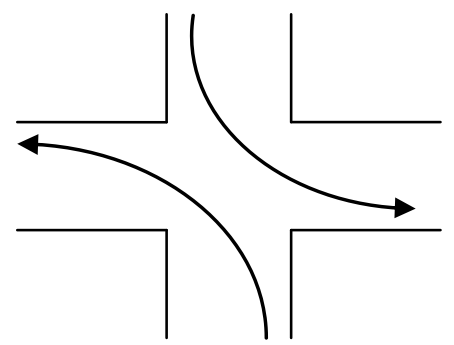

(d) phase-4

Figure 2. 4-Phase Control System of Crossroad

\subsection{Modeling}

By modeling a crossroad traffic control system based on the Extended Colored Petri Nets with The Inhibitory and Enable Arc, it can realize the detection and adjustment of "green conflict". According to the actual traffic condition, we set west-east direction as the trunk road in the model, i.e., once "green conflict" appears at the west-east direction and north-south direction, the west-east direction have crossing priority after adjustment. When the "green conflict" of the system appears, the light of corresponding crossroad could be yellow flickering. If the system can return to normal, then all lights become red and the system comes back to the initial phase (i.e., phase 1). In order to simplify the model and fit reality, the "green conflict" of the same crossroad caused by equipment hardware failure is not considered here. Figure 3 shows model of the system. 


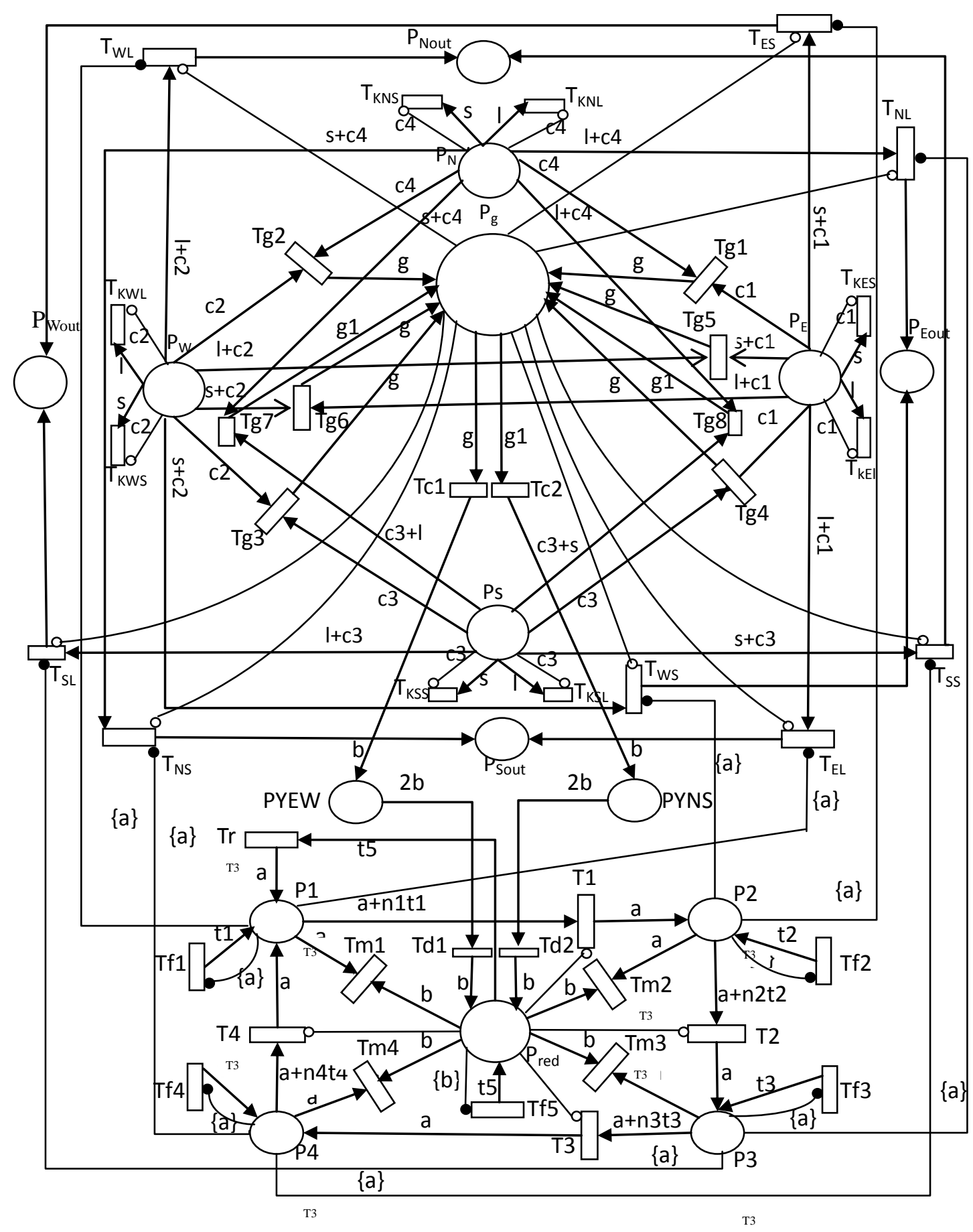

Figure 3. Model of Detecting "Green Conflict"

In the model, $P_{E}$ represents the state of the signal light at crossroad east, its color set is $C=\{S, L, C 1\}, S$ and $L$ respectively represents the green light signal of straight-on and left-turn at crossroad east, while $C l$ shows that green light at crossroad east is on. The transition $T_{E S}$ and $T_{E L}$ signify that the vehicles are running straight-on or left-turn at crossroad east; $P_{\text {Eout }}$ means that the vehicles are allowed to leave from the crossroad east; $P g$ represents the Place of "green conflict"; $T g 1 \sim T g 8$ are used to detect whether there is "green conflict" in the system, the Place $P g$ has a color value $g$ or $g 1$ if one of $T g 1 \sim T g 8$ occurred, so this result means that "green conflict" happened. PYEW and PYNS respectively stand for signal of yellow light flickering at west-east direction and 
north-south direction of the crossroad, their color set is $C=\{b\}$. The Place Pred represents the all-red signal of the system. $P 1, P 2, P 3$ and $P 4$ respectively indicate the occurring permission authority of the four phases, the system runs this phase if the Place has a color a. $\operatorname{Tr}$ represents the initial transition, the system would come back to the initial phase once it occurred. $T c 1, T c 2$ represent the control signals of yellow flickering, they occurred when "green conflict" is detected in the system, then the Place PYEW and PYNS have the color $b$, the color $b$ stands for the color of transition which control green conflict processing. $T d l$ and $T d 2$ are Transitions which deal with "green conflict". $T d l$ will occur firstly when the token of PYEW accumulates to $2 b$, and then the Place Pred will have the color $b$, and the system run into the state of all-red. At the same time, on one hand one of Tm1 Tm4 occurred, on the other hand it makes Tf5 able. When one of Tm1 Tm4 occurred, the token of corresponding Place Pl P4 is removed, namely, the current system's phase is wiped off; when the Transition Tf5 occurred, the token of the Place Pred could add a color $t 5$, this color would enable $T r$ to occur and make the system come back to the initial state. TfI Tf4 represent a clock pulse generator which is controlled by central control system. Transition T1,T2,T3 and T4 mean that the changing between each phase. Under normal circumstances (no "green conflict" happen), that Place $P l$ owns token $a$ means running the phase 1 , so $T_{f l}$ occurred. At every $t l$ time interval, transition $T c l$ will occur one time and token in Place $P 1$ will be added $t 1$. After time interval of $(n 1 * t 1)$, the token in $P 1$ would change to $\left(n l^{*} t 1+a\right)$. At the same time, $T 1$ is enabling. After $T 1$ has been triggered, $P 1$ lose the token $a$; and $P 2$ get the token $a$. So the system will run into phase 2 .

\subsection{Model Simulation}

Basing on logical equivalence principle, we use the Verilog HDL to describe the above-mentioned model. The input signals of the system are PEinw, PSinw, PWinw and PNinw, they respectively represent the state of signal lights in the east-crossroad, south-crossroad, west-crossroad and north-crossroad, they are stored by a 24-bit register respectively, every 4-bit is as a unit and it is divided into six parts. The six units store $C 4$ $C 3 C 2 C 1 S L$ from $M S B$ to $L S B$. Among them, $C 1, C 2, C 3$ and $C 4$ respectively represent green-light signal in the east-crossroad, west-crossroad, south-crossroad and north-crossroad, while $L$ and $S$ represent that the green-light signal for straight-on and left-turn are on. The output signal of the system are "green conflict" signal (Pgout), the yellow flickering signal of west-east direction (PYEWtoken), the yellow flickering signal of north-south direction (PYNStoken), the all-red signal (Predout) and the current phase signal of the system (Pnout) respectively. Among them, that the first-bit of Pnout is 1 indicates that the current system is running in phase 1, that the second-bit of it is 1 show that the system exists in phase 2, and so on. The simulation result of the overall operation of the system is shown in Figure 4.

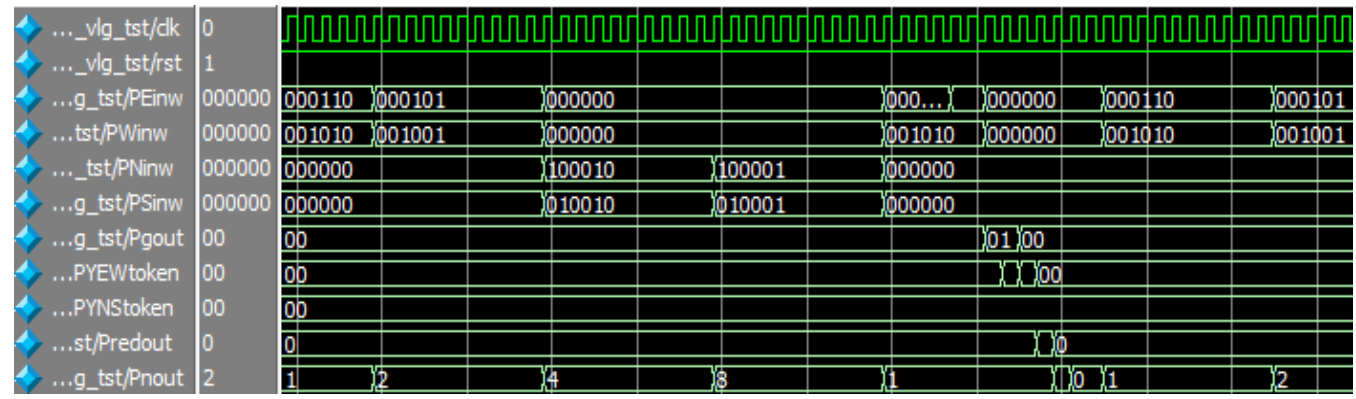

Figure 4. The Simulation Result of the Overall Operation of the System 
From Figure 4, when the system begin to run, PEinw equals to 000110 and PWinw equals to 001010 , these mean that the current system is running in phase 1 and the green-light stands for west-east direction straight-on. In the following period of time, if there is no "green conflict", the system turns sequentially from phase 1 to the next state. But during the phase 1, if PEinw equals to "000101" (allowing left-turn in the east-crossroad) and PWinw equals to "001010" (allowing straight-on in west-crossroad), this means that the "green conflict" occurred in the system. Soon afterwards, the system detected the phenomenon, now Pgout equals to 1 , the yellow flickering signals turn on, and then the system come into all-red mode. After a period of adjustment, the system comes back to the initial phase (phase 1).

In order to analyze the simulation result clearly, we divide the picture into two sections. The first part is the normal running state which is shown in Figure 5. The second part is the operation state of "green conflict", as shown in Figure 6

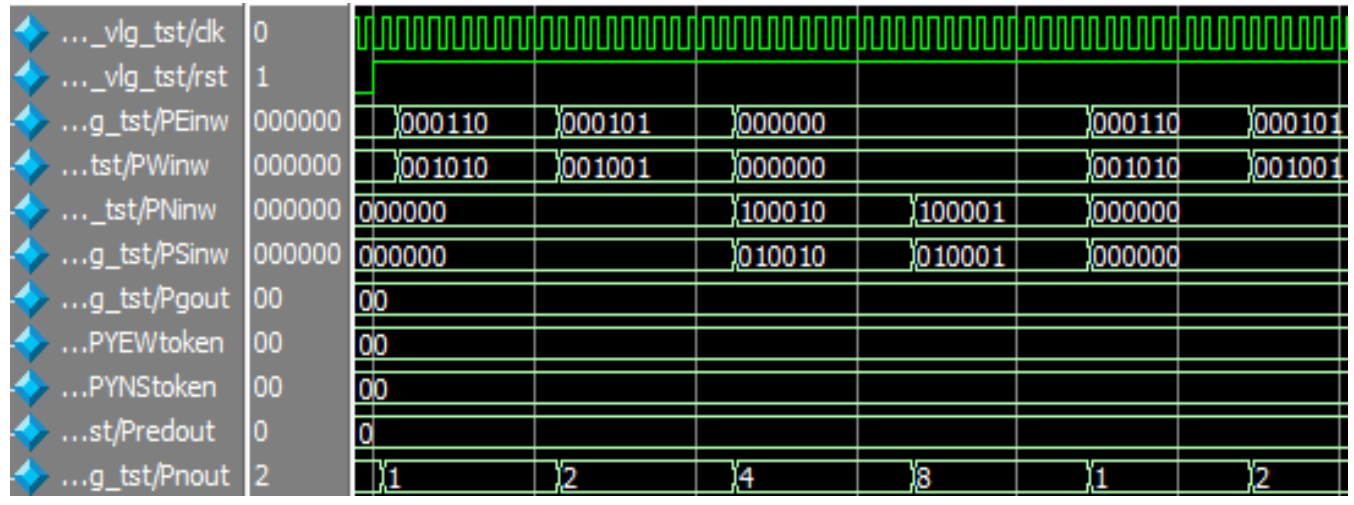

Figure 5. The Simulation Result on Normal Circumstance

After the reset signal, the model begins to run. Firstly, that Pnout equals to 1 denotes that the current system is running in the phase 1, at the same time Pgout, PYEWtoken, PYNStoken, Predout are equal and their value equal to 0 , that is to say there is no "green conflict", therefore the system is running in the normal state. Four phases alternated sequentially, and each phase runs 10 clock cycles.



Figure 6. The Simulation Result of "Green Conflict"

From Figure 6, We can see occurrence of "green conflict" during the phase 1 and Pnout equals to 1, namely, that PEinw equals to 000101 means that the green signal of left-turn direction at east-crossroad is on , and that PWinw equals to 001010 means that the green signal of straight-on direction at west-crossroad is on. After two clock cycles, that Pgout equals tol means that the system has already detected the phenomenon of "green conflict", so the system begin to adjust automatically. The signal PYEWtoken and Predout shows the concrete adjustment process, that PYEWtoken don't equal to 0 means yellow 
flickering, and soon that Predout equals to 1 means all-red mode, so the vehicles do not be allowed to pass the two direction. During the adjustment stage of "green conflict", the phases of the system no longer change alternately. Finally, the system perhaps will come back to the initial phase by fault processing module; and turn into the normal operating state at last.

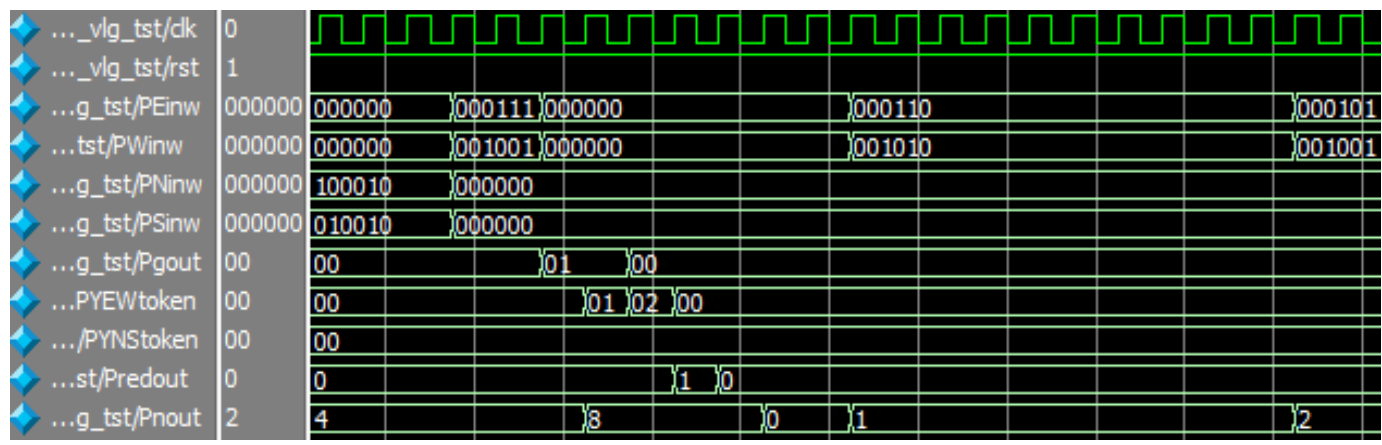

\section{Figure 7. The Simulation Result of "Green Conflict on Asymmetric Phase"}

From Figure 7, We can see occurrence of "green conflict" during the phase 3 and Pnout equals to 4, namely, that PEinw equals to 000111 means that the green signals of straight-on and left-turn direction at east-crossroad is on , and that PWinw equals to 001001 means that the green signal of left-turn direction at west-crossroad is on. After two clock cycles, that Pgout equals to 1 means that the system has already detected the phenomenon of "green conflict" ("Asymmetric Green Conflict"), so the system begin to adjust automatically. The signal PYEWtoken and Predout shows the concrete adjustment process, that PYEWtoken don't equal to 0 means yellow flickering, and soon that Predout equals to 1 means all-red mode, so the vehicles do not be allowed to pass the two direction. During the adjustment stage of "green conflict", the phases of the system no longer change alternately. Finally, the system perhaps will come back to the initial phase by fault processing module; and turn into the normal operating state at last.

\section{Conclusion}

In this paper, an Extended Colored Petri Nets with The Inhibitory and Enable Arc based on the Classics Petri Nets is presented, it takes advantage of Color Petri Nets to describe the control information of the system; the inhibitory-arc enhances the modeling capability and simplifies the model. This kind of method can not only describe the complex system but also make the mode more intuitive. We used it to build a traffic control model of the urban crossroad, and transform it into Verilog HDL description form. Finally, we verify the reliability of the model and achieve the goal as expected, .i.e., it can reflect the traffic flow on normal circumstance, and detect and adjust the "green conflict" on abnormal circumstance.

In the future we will focus on the model-level division based on the Extended Colored Petri Nets with The Inhibitory and Enable Arc, and we intend to do more research on reliable, multi-functional and adaptive mode of regional traffic control system.

\section{Acknowledgments}

The project was supported by the key item of Sichuan Province Education Department (NO.13ZA0032) and Xihua university item YJD20140005. 


\section{References}

[1] X. Liu and G. Wen. "Modeling and Analysis of Traffic Control System Based On Petri Nets". Computer Engineering, vol. 34, no. 09, (2008), pp.8-10.

[2] Y. Bu and D. Li, "The Research on the Urban Traffic Signal Control System Based On the Petri Nets", The Information Technology of ChengDu University, vol. 24, no. 3, (2009), pp. 244-249.

[3] L. Wells, "Performance analysis using CPN tools", Proceedings of the $1^{\text {st }}$ international conference on Performance evaluation methodologies and tools, ACMM Press, (2006).

[4] J. Li and H. Zhao, "Modeling and Analysis of Traffic Information Grid System Based On Color Petri Nets", Microcomputer Information, vol. 27, no. 6, (2011), pp. 20-21.

[5] M. Azzumar, A. Halim and M. Harjono, "Performance evaluation of two ways urban traffic control system based on macroscopic hybrid petri net model", ICACSIS, (2013), pp. 335-340.

[6] Y.-S. Huang, Y.-S. Weng, J. M. Der and B.-Y. Chen, "Based on synchronized timed Petri nets for urban traffic control systems". IEEE, SMC, (2013), pp. 384- 390.

[7] S. Liu and X. Le, "Modeling and Behaviour Analysis on the Petri Nets with Inhibitory-arc and Test-arc", Journal of System Simulation, vol. 20, (2009), pp. 76-89.

[8] C. Lin, Z. Yang and B. Gong, "Dynamic model of transit signal priority on colored time petri net", ICCTP, (2009), pp. 1-7.

[9] B. Li, B. Ma, T. Tang and K. Chen, "The Research on Verification Method of Embedded System Model Based on TCPN", AISS, vol. 4, no. 23, (2012), pp. 207-214.

[10] B. Li, B. Ma, T. Tao and K. Chen, "The Research of Embedded Systems Modeling Based on OOTCPN", AISS, vol. 4, no. 23, (2012), pp. 299-305.

[11] B. Li, T. Tang, K. Chen and Y. Li, "Modeling and Analysis of Embedded Systems Based on Extended Colored Petri Nets", IJACT, vol. 4, no. 23, (2012), pp. 546-551.

[12] Dotoli, Mariagrazia, Fanti and M. Pia, "An Urban Traffic Network Model via Coloured Timed Petri Nets", CONTROL ENGINEERING PRACTICE, vol. 14, (2006), pp. 1213-1229.

[13] M. A. Badamchizadeh and M. Joroughi, "Deterministic and Stochastic Petri Net for Urban Traffic Systems", ICCAE, vol. 5, (2010), pp. 364-368.

[14] Y.-S. Huang, Y.-S. Weng and M. Zhou, "Modular Design of Urban Traffic-Light Control Systems Based on Synchronized Timed Petri Nets", IEEE, vol. 15, (2014), pp. 530-539.

[15] Ng, Kok Mun, Reaz, M. B. Lbne and A. M. A. Mohd, "A Review on the Applications of Petri Nets in Modeling Analysis and Control of Urban Traffic", IEEE TRANSACTIONS ON INTELLIGENT TRANSPORTATION SYSTEMS, vol. 14, (2013), pp. 858-870.

[16] J. Julvez, and R. K. Boel, "A Continuous Petri Net Approach for Model Predictive Control of Traffic Systems", IEEE TRANSACTONS ON SYSTEMS MAN AND CYBERNETICS PART A-SYSTEMS AND HUMANS, vol. 40, no. 4, (2010), pp. 686-697. 
International Journal of Signal Processing, Image Processing and Pattern Recognition Vol.8, No.8 (2015) 